\title{
The legacy of AGPS
}

By Sharif Islam, Semi-retired Associate, London, UK

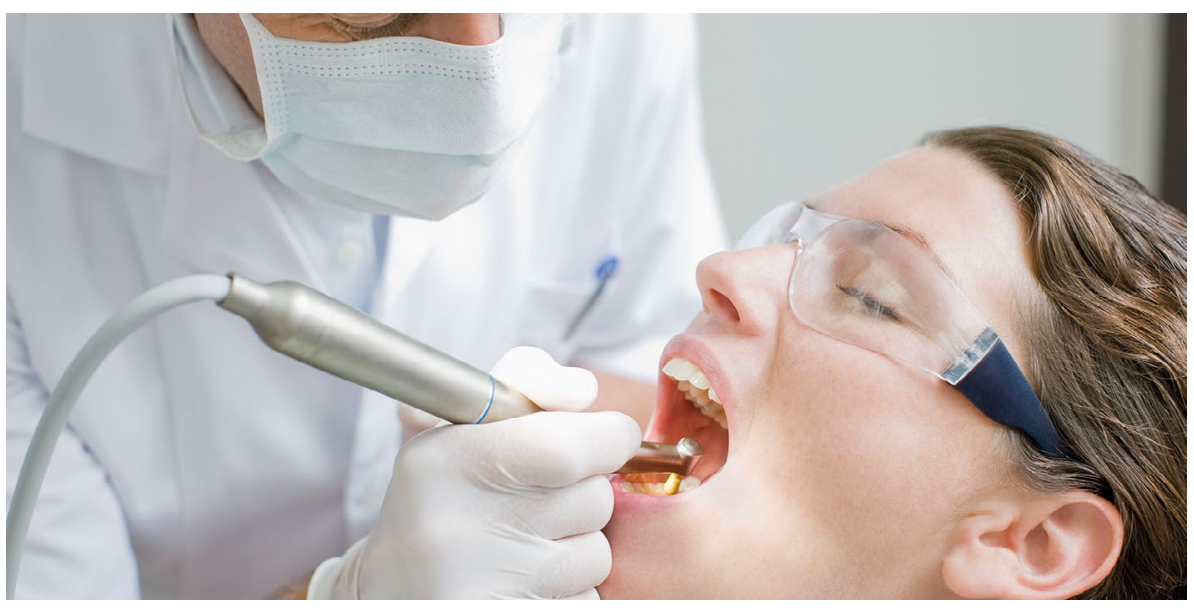

W

e're all becoming extremely proficient at using a slow and speed-reducing hand-piece, say my esteemed colleagues, which is the legacy in a nutshell.

But there's more to it than that.

The desperate aversion to blankets of PPE and slow suffocation inside an FFP3 mask has incentivised a great deal of creativity in operative dentistry. No longer do we nonchalantly grab the high-speed drill to slice through enamel and old amalgams while waterboarding the patient as the aspirator catches up. The days of recoiling in despair at the mid-morning filling appointment and its post-operative fallow time are fast becoming history. That oppressive rubber mask is safely entombed in its sterile box, peeking out and yearning for release but firmly denied by a practitioner determined not to succumb.

Granted, there are some procedures where an aerosol is unavoidable and irrigation of the drill tip is essential. Preparing a tooth for a crown, for example, already carries a risk of pulp death even with cool water bathing the cut. Even if preparing a non-vital tooth it's a foregone conclusion that the patient would still prefer not to smell themselves burning.

But for the restoration of small and mediumsized cavities many practitioners have resorted to a slow and intermittent entry with a greatly reduced spin of the bur. So gentle, indeed, that no heat is generated and often no pain is felt.
Small deciduous cavities can be cleaned with gentle excavation by hand or slow-speed rotary burs, negating the use of any anaesthetic and thus winning the approval of a nervous child.

The technique is not quite the atraumatic restorative technique (ART) employed with limited facilities in developing nations. While the rationale for starving cariogenic bacteria, and thus arresting caries progression, by sealing them off with restorative material is sound, most practitioners have the aim and the means to remove as much of the caries as possible.

Local anaesthetic is always a good idea, however, when venturing into the vicinity of an adult pulp, as it is usually oblivious to one's noble intention to remove the disease around take longer than a typical restorative procedure with high-speed drilling, but one suspects far less time than it takes to mummify oneself in a gown and gas mask. Effective aspiration is still necessary, of course, lest particles of debris should navigate past the tonsils or worse still, onto the patient's expensive clothes.

Even our esteemed hygienists have found that the prohibition of ultrasonic scaling, although much to the chagrin of many patients, has enabled more focused debridement and better tactile feedback. The old maxim that when you employ a machine to do the work of a human, you take something away from the human, may be worthy of merit.

In time, some of us may revert to a traditional pace of drilling and filling with as much alacrity as liberation from PPE will allow. But for many of us a peculiar, even Aristotelian, confluence of events may have etched a lasting legacy on the future of operative dentistry. The transformation of aerosol generating procedures into aerosol avoidance procedures has made our patients more grateful for not losing parts of their face to local anaesthetic for extended periods, while collecting less water in their airways and less noise in their eardrums. And, of course, practitioners are more grateful for not being cocooned in multiple layers of insulating plastic. Even after the pandemic protocols are lifted, dentists may continue

\section{'The old maxim that when you employ a machine to do the work of a human, you take something away from the human, may be worthy of merit.'}

it. For the nerve, having any bur foraging inside the cavity at any speed is probably akin to standing next to a Bertha tunnel boring machine as it excavates solid granite inside a mountain. Not a pleasant vibration, let's agree. Thus, sound judgement should be exercised when using a dry bur, weighing that against the potential for possible pulpal irritation.

Mitigating said irritation, a delicate touch may therefore offset post-operative pain. It may to exercise a measured approach to cavity design, one that is potentially less painful, less destructive and informed by minimal intervention.

However long we are compelled to use PPE and fallow times, it is likely that the aftermath of aerosol generating procedures will host many changes and encourage us to advance and adapt the practice of dentistry in the postpandemic era. 